\title{
Is the subharmonic threshold always lower than the fundamental one in plane Poiseuille flow?
}

Cite as: Phys. Fluids 30, 014103 (2018); https://doi.org/10.1063/1.5011088

Submitted: 30 October 2017 . Accepted: 02 January 2018 . Published Online: 24 January 2018

Rangachari Kidambi (iD), and Usha Srinivasan

\section{ARTICLES YOU MAY BE INTERESTED IN}

Optimum-wavelength forcing of a bluff body wake

Physics of Fluids 30, 015101 (2018); https://doi.org/10.1063/1.4999091

Manipulation and control of instabilities for surfactant-laden liquid film flowing down an inclined plane using a deformable solid layer

Physics of Fluids 30, 014104 (2018); https://doi.org/10.1063/1.5008292

Aerodynamic heating in transitional hypersonic boundary layers: Role of second-mode instability

Physics of Fluids 30, 011701 (2018); https://doi.org/10.1063/1.5005529

\section{Scilight Hightights of the best new research} in the physical sciences 


\title{
Is the subharmonic threshold always lower than the fundamental one in plane Poiseuille flow?
}

\author{
Rangachari Kidambi ${ }^{\mathrm{a})}$ and Usha Srinivasan ${ }^{\mathrm{b})}$ \\ Computational and Theoretical Fluid Dynamics Division, National Aerospace Laboratories, \\ Bangalore 560017, India
}

(Received 30 October 2017; accepted 2 January 2018; published online 24 January 2018)

\begin{abstract}
We revisit the secondary instability of a Tollmein-Schlichting wave in plane Poiseuille flow at $R e=$ 5000 and find that the fundamental threshold can be lower than the subharmonic one in the wavenumber space, in contrast to the prevalent view in the literature that only instability of subharmonic modes occurs at very low disturbance amplitudes and fundamental modes need a higher disturbance amplitude to become unstable. This is because of overlooking the even class of fundamental modes, which happen to produce the lower threshold at those wavenumbers. Published by AIP Publishing. https://doi.org/10.1063/1.5011088
\end{abstract}

\section{INTRODUCTION}

Secondary instability has long been considered a key mechanism in explaining subcritical transition in wallbounded shear flows. ${ }^{1}$ A variety of base states have been considered for the linearisation-the dominant TollmeinSchlichting (TS) mode with the damping neglected, ${ }^{2}$ nonlinear equilibria and quasi-equilibria, ${ }^{3}$ and streamwise vortices and streaks. ${ }^{4}$ Typically, in all such studies, the base state has to be considered in a reference frame moving with an appropriate velocity. This renders the coefficients of the disturbance equations periodic in the frame variable with the implication that Floquet modes, in that variable, can be sought. The general strategy is to study temporal secondary instability; a basic wavenumber $\alpha$ is prescribed, and the secondary temporal growth rate determined from the solution of an eigenvalue problem. The spatial problem, arguably more relevant to disturbance evolution in such flows but also more complicated to solve, is not pursued often, for good reason; Herbert et al. ${ }^{5}$ have shown how to calculate secondary spatial growth rates from the temporal ones.

Reference 2 was a pioneering study of, mainly, the subharmonic instability of the least stable mode in plane Poiseuille flow (pPf, hereafter) at $R e=5000$, where the Reynolds number is defined in the usual way, based on the half-height of the channel. Since then, a general view that the subharmonic instability dominates at low primary disturbance amplitudes, often called the N-regime, has been prevalent in the literature [e.g., Yaglom and Frisch ${ }^{6}$ (p. 501ff.)]. In this work, we re-investigate the secondary instability of the least stable mode to 3D disturbances in plane channel flows and find that this view may not be entirely correct. In fact, we find that there are parameter regimes for which the fundamental mode (defined in Sec. II), not the subharmonic, has the lower threshold amplitude.

The goals of the paper are two-fold: (i) to provide extensive numerical data, of eigenvalue spectra and threshold

a)Electronic mail: kidambi@ctfd.cmmacs.ernet.in

b)Electronic mail: usha@ctfd.cmmacs.ernet.in amplitudes, for the secondary instability of the least stable mode for the canonical case of $\operatorname{Re}=5000$, considered by Ref. 2 , and (ii) to document the existence of a class of solutions that seem to have been overlooked in the literature. The paper is organised as follows. Section II sketches the well-known problem formulation and solution. Extensive numerical results for a wide range of parameters and comparisons with published numerical and experimental data are presented in Sec. III. We discuss the implications of the fact that the new class of fundamental solutions can have lower disturbance thresholds for experimental observations in Sec. IV.

\section{FORMULATION AND SOLUTION}

Following Herbert, ${ }^{2,5}$ we assume the base flow for the secondary analysis to be composed of plane Poiseuille flow and a traveling wave $A f\left(x-c_{r} t\right) ; A$ and $c_{r}$ are, respectively, the amplitude and real phase velocity of the wave. A sketch of the base flow for the secondary analysis is shown in Fig. 1. The phase velocity and wavenumber $\alpha$ are those of the least stable mode, the so-called TS wave, obtained from the primary linear stability analysis of the plane Poiseuille flow. The stream function of the periodic base flow is given by

$$
\psi(y, \xi)=\Psi(y)+A \phi(y) e^{i \alpha \xi}+\text { c.c. },
$$

where $\Psi(y)=y-y^{3} / 3, \xi=x-c_{r} t$, and $\phi(y)$ is the eigenfunction associated with the principal mode of the Orr-Sommerfeld (OS) equation.

The evolution equations for the disturbance are obtained by substituting the total velocity

$$
\vec{v}(\xi, y, z, t)=\vec{v}_{2}(\xi, y)+\epsilon \vec{v}_{3}(\xi, y, z, t)
$$

in the Navier-Stokes equations; $\vec{v}_{2}$ is the $2 \mathrm{D}$ velocity of the base flow that is obtained from $\psi(y, \xi)$, and $\epsilon \vec{v}_{3}$ is the perturbation velocity. The resulting disturbance equations, when linearized in $\epsilon$, have coefficients periodic in $\xi$. These equations are given, for example, in the study of Herbert et al. ${ }^{5}$ and are reproduced in the Appendix, for completeness. Standard Floquet theory ${ }^{7}$ indicates that the disturbance equations, with 

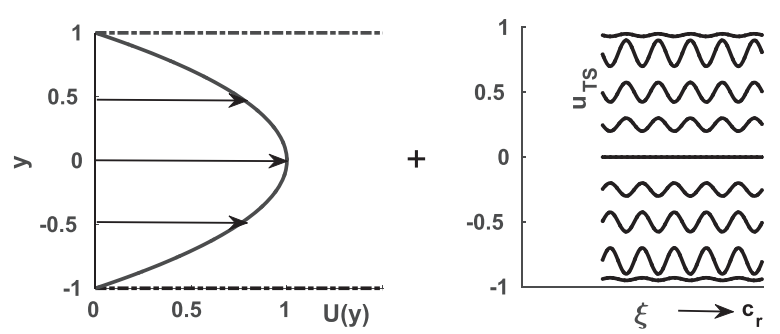

FIG. 1. Schematic of the periodic base state. Fully developed Poiseuille flow profile $U(y)$ is shown on the left. The figure on the right-hand side represents the periodic TS disturbance added to $U(y)$ at different $y$ locations; the travelling wave is shown in a reference frame moving with speed $c_{r}$. For simplicity, only the streamwise disturbance component is shown.

periodic coefficients of period $\lambda=2 \pi / \alpha$, admit solutions of the form

$$
\left[\begin{array}{l}
u_{3}(\xi, y, z, t) \\
v_{3}(\xi, y, z, t)
\end{array}\right]=e^{\gamma \xi} e^{\sigma t} e^{i \beta z} \sum_{m=-\infty}^{\infty}\left[\begin{array}{l}
u_{m}(y) \\
v_{m}(y)
\end{array}\right] e^{i m \alpha \xi}
$$

where $\beta$ is the spanwise wavenumber; $\gamma$ is the Floquet or characteristic exponent and can range from 0 to $i \alpha / 2$, the ends corresponding to fundamental and subharmonic modes, respectively. The reason for these names can be seen by considering the Floquet or characteristic multiplier defined as $\rho=e^{\gamma \lambda}$. Given that

$$
\vec{v}_{3}(\xi+\lambda, y, z, t)=\rho \vec{v}_{3}(\xi, y, z, t),
$$

it is easy to see that the fundamental and subharmonic modes correspond to $\rho= \pm 1$, with wavelengths $\lambda$ and $2 \lambda$, respectively. The former arise from fundamental resonance in the parametrically excited system, whereas the latter are as a result of the principal parametric resonance. Complex $\rho$ correspond to detuned resonances. A good, concise review can be found in the study of Yaglom and Frisch ${ }^{6}$ (p. 493ff.).

As mentioned by Herbert, ${ }^{2}$ the functions $f_{m}(y)$, given by

$$
f_{m}(y)=\left[\begin{array}{l}
u_{m}(y) \\
v_{m}(y)
\end{array}\right],
$$

are alternatingly even and odd when the principal streamfunction is even. As is well known, the least stable mode in pPf has even streamfunction. For an odd principal streamfunction, it can be seen that $f_{m}(y)$ are all even or odd, and these correspond to different eigenvalue families. Further, it is also known that the streamwise and transverse velocity components at each $m$ level, $u_{m}$ and $v_{m}$, are also of opposite parity. Using this fact, the eigenspectrum for any $\gamma$ can be split into two disjoint sets: $\mathcal{E}$ and $\mathcal{O}$, where $\mathcal{E}$ (respectively, $\mathcal{O}$ ) represents the set of eigenvalues for which $u_{0}$ is even (respectively, odd). Hereafter, the Floquet modes belonging to these sets will be called even and odd modes, respectively, with the understanding that only the dominant Fourier component of the modes is even or odd.

With the ansatz (3), the system of PDEs (AI) becomes an ODE system in the normal coordinate $y$. Standard Chebyshev collocation (e.g., Schmid and Henningson ${ }^{4}$ ) renders this into a matrix eigenvalue problem, which is then solved by standard routines. We avoid repetition of the well-known details and present the results.

\section{RESULTS}

Unlike the primary spectrum, the secondary spectrum for pPf does not seem to have been often computed; only Herbert (see Fig. 2 of Ref. 2) presents the spectrum for one case and other derived quantities like growth rates of the dominant mode and threshold amplitudes for another. Chebyshev collocation with 18 points in a half channel was used in that computation. The $m=-1,0$ modes were used in the subharmonic case, while these were supplemented with the $m=1$ mode in the fundamental case. No results for detuned resonances were presented.

In what follows, we provide a detailed comparison with Herbert's ${ }^{2}$ results. We first compare the eigenspectra, then the threshold amplitudes and finally the disturbance velocity profiles.

\section{A. Comparison of eigenspectra}

We use the same parameter values as in Ref. $2-R e$ $=5000, \alpha=1.12, \beta=2, A=0.0248$. Both fundamental and subharmonic modes will be considered. For a fair comparison, we first compute with 40 collocation points in the full channel and also with 20 points in the half channel. We also retain the same $m$ modes as Herbert. ${ }^{2}$ Later, we will present a convergence study with a varying number of Fourier modes $M$ and Chebyshev modes $N$.

Figure 2 shows the normalised primary TS eigenfunction (triangles) that has been used in the computation of the Floquet modes for $R e=5000$ and $\alpha=1.12$. The eigenfunction was normalized such that the maximum absolute value attained by the complex streamwise velocity is unity. For comparison, Herbert's velocity distribution (from Fig. 5 of Ref. 2) is plotted as circles. There is a small discrepancy between the two, the reason for which could be a difference in the distribution of points in the two computations (more on this below). The collocation points used in the present study are Gauss-Lobatto (G-L) points.

$\mathcal{E}$ and $\mathcal{O}$, for the subharmonic mode, have been computed using $100 \mathrm{G}-\mathrm{L}$ points across the half-channel by incorporating

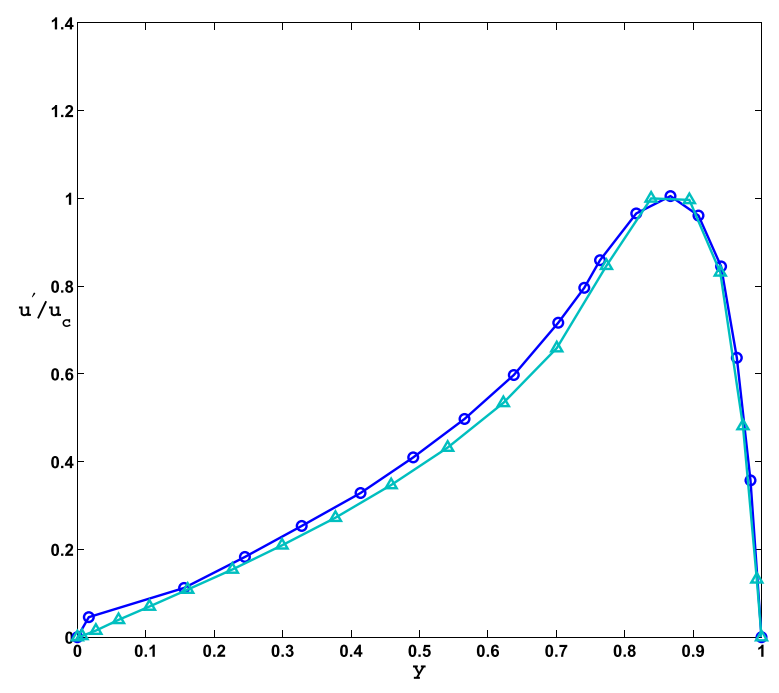

FIG. 2. Normalized principal streamwise velocity across half channel width; $R e=5000, \alpha=1.12$. Blue circles: Fig. 5 of Ref. 2 with 18 collocation points; green triangles: present computation with $20 \mathrm{G}-\mathrm{L}$ points. 


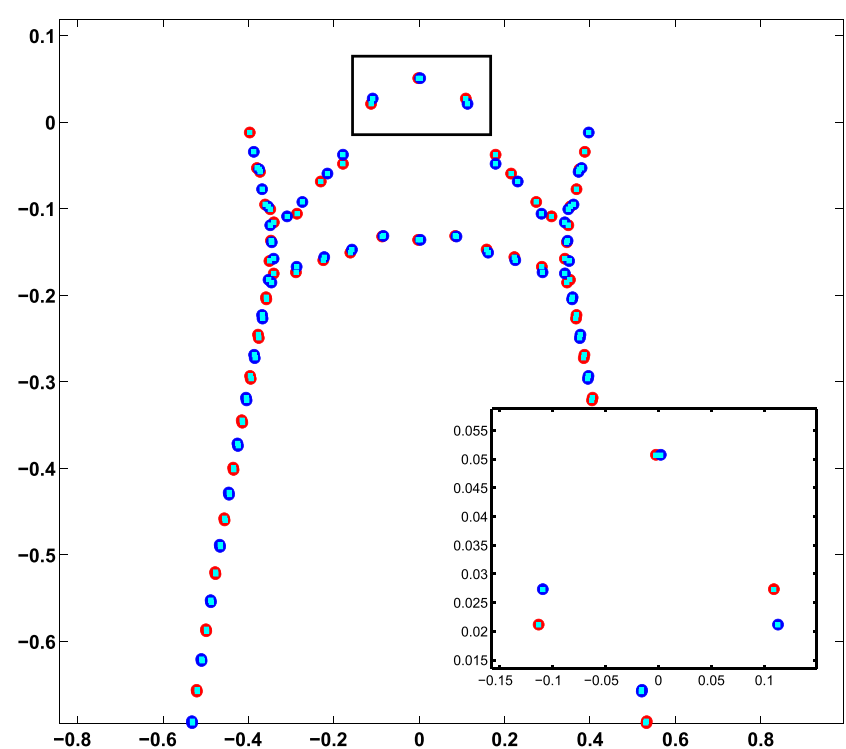

FIG. 3. Eigenspectrum of subharmonic disturbances for $R e=5000, \alpha=1.12$, $\beta=2.0$, and $A=0.0248$. Red and blue circles indicate half channel computations, using $100 \mathrm{G}-\mathrm{L}$ points, with parity boundary conditions. Cyan squares represent a full channel computation with $200 \mathrm{G}-\mathrm{L}$ points.

the appropriate parity conditions at the channel centreline. Alternatively, we also computed the spectrum with $200 \mathrm{G}-\mathrm{L}$ points for the full channel. The half and full spectra are shown in Fig. 3 and are in good agreement.

A comparison with Herbert's ${ }^{2}$ spectrum (Fig. 2 of that paper) for the same case appears in Fig. 4. Herbert's spectrum contains only half the number of eigenvalues as that of the present computation because he computes only one set of eigenvalues, $\mathcal{O}$. The eigenspectrum of Ref. 2 does not show any symmetry, but interestingly the present computation shows symmetry about the imaginary axis. In fact, one half of the present spectrum matches closely with that of Ref. 2, and the

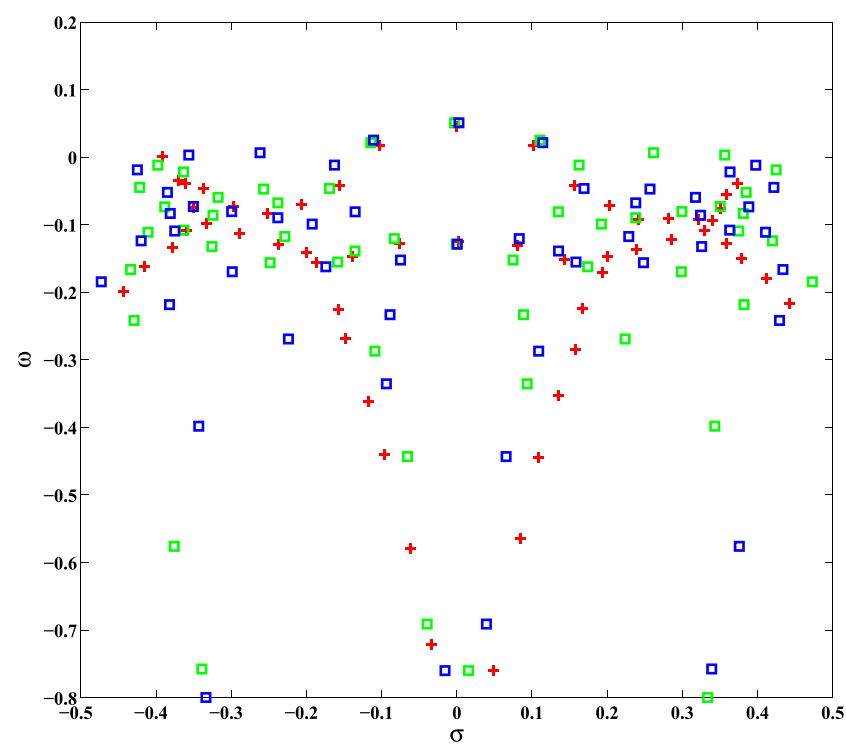

FIG. 4. Eigenspectrum of subharmonic disturbances at $R e=5000, \alpha=1.12$, $\beta=2.0$, and $A=0.0248$. Dark and light squares denote half channel computations using 18 collocation points while the red + represent data from Fig. 2 of Herbert. ${ }^{2}$ remaining half of the spectrum is placed symmetrically to that of Ref. 2. As stated earlier, the Floquet eigenspectrum splits into two disjoint sets; though each of these sets is asymmetric, the total spectrum is symmetric.

Only the lowest few eigenvalues show satisfactory agreement. The major reason for the non-matching of the other eigenvalues is the lack of convergence of the higher modes (in Ref. 2 as well as the present computation) when only 20 G-L points are used. The convergence of the eigenvalues seems to be related to the number of collocation points resolving the critical layer. Thus, Herbert ${ }^{2}$ obtains a well-formed spectrum even with 18 grid points in a half-domain computation as his collocation points are concentrated more near the critical layer than near the centre of the channel (blue circles in Fig. 2). In contrast, the present half-domain computation with 20 G-L collocation points yields a relatively poorly structured spectrum as a large percentage of the points is concentrated near the channel centre (green triangles in Fig. 2). The least stable eigenvalues, however, converge and also match those of Ref. 2. Computations were also performed with 19 and 21 collocation points and large variations in all but the least stable eigenvalues was found, confirming that more points are required for a converged spectrum.

A full channel computation using $40 \mathrm{G}-\mathrm{L}$ points was done as well; the comparison is shown in Fig. 5. A larger percentage of these 40 points are clustered around the critical layers, and hence this computation shows a better structured spectrum and is closer in appearance to that in Ref. 2. The least stable eigenvalue of the present computation $\sigma=0.00178+$ $i 0.0409$ is $O\left(10^{-3}\right)$ different from that of Ref. $2(\sigma=0.002+$ i0.0465). Herbert ${ }^{2}$ assumes an $e^{\omega t}$ dependence, whereas we assume, in accordance with standard practice, an $e^{i \omega t}$ dependence. Hence, for comparison, we have multiplied Herbert's values by $\sqrt{-1}$. There is also another eigenvalue symmetric to $\sigma$, at $-0.00178+i 0.0409$, but Ref. 2 does not compute this.

Figure 3 of Ref. 2 shows the variation of the amplification rate and frequency for the dominant mode of subharmonic

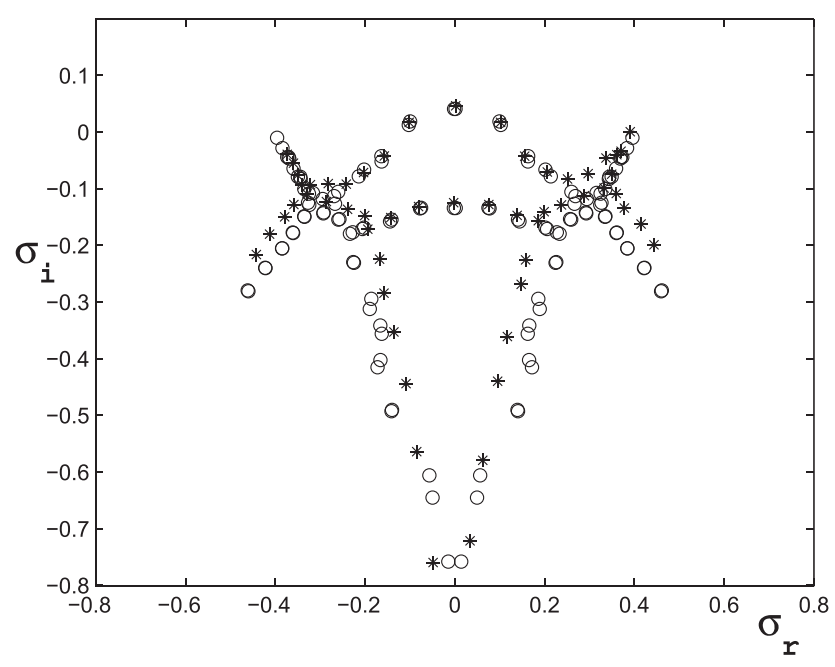

FIG. 5. Eigenspectrum of subharmonic disturbances at $R e=5000, \alpha=1.12$, $\beta=2.0$, and $A=0.0248$. Circles: full channel computations using $40 \mathrm{G}-\mathrm{L}$ points; stars: Fig. 2 of Herbert. ${ }^{2}$ 


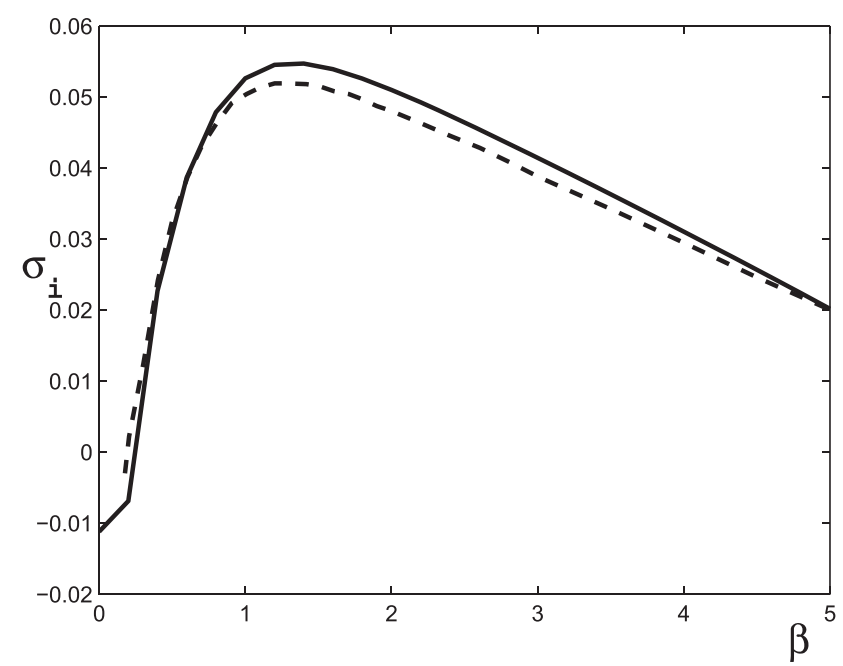

FIG. 6. Amplification rate for the dominant mode of subharmonic instability as a function of $\beta$ at $\operatorname{Re}=5000$ and $\alpha=1.12$. Solid and dashed curves represent present and Herbert's ${ }^{2}$ results.

instability as a function of $\beta$ at $R e=5000$ and $\alpha=1.12$. We provide a comparison with the present calculation in Fig. 6. The same $O\left(10^{-3}\right)$ error that was noted earlier persists. However, the curves are similar in shape with maxima roughly occurring at similar $\beta$.

On p. 873 of Ref. 2 (Fig. 4), the amplification rate $\sigma$ of the dominant mode of subharmonic instability, for the case $\alpha=1.02, \beta=2$, is presented as a function of $A$. The accompanying text states that the dominant eigenvalue $s_{1}$ tends to $-0.0225-i 0.0626$ as $A \rightarrow 0$.

We present the converged spectrum for the same case in Fig. 7, for $A=0 . M=3$, and $N=200$. As noted in the figure, there is an eigenvalue $\tilde{s}_{1}=-0.0205-i 0.0655$ which is different by $O\left(10^{-3}\right)$ from Herbert's value. However, it is not the dominant eigenvalue, as apparent from the figure; the dominant ones are also indicated in the figure and are located at \pm 0.365 $-i 0.008$. However, for $A \approx>0.004$, the dominant eigenvalue is the same one depicted in Fig. 4 of Ref. 2.

Figure 8 shows the variation of amplification rate and frequency of the dominant subharmonic mode as a function of the amplitude $A$; the corresponding curve from Fig. 4 of Ref. 2

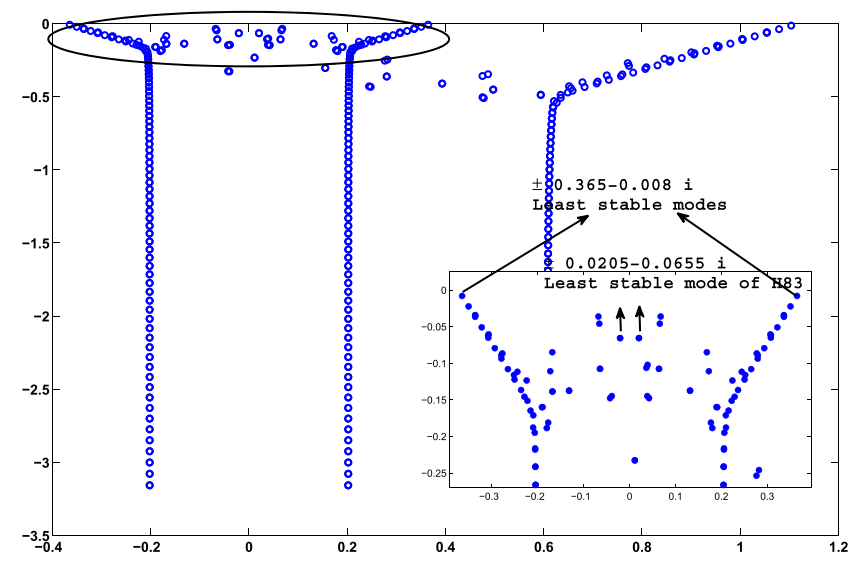

FIG. 7. Eigenspectrum of subharmonic disturbances at $R e=5000, \alpha=1.02$, $\beta=2.0$, and $A=0$.

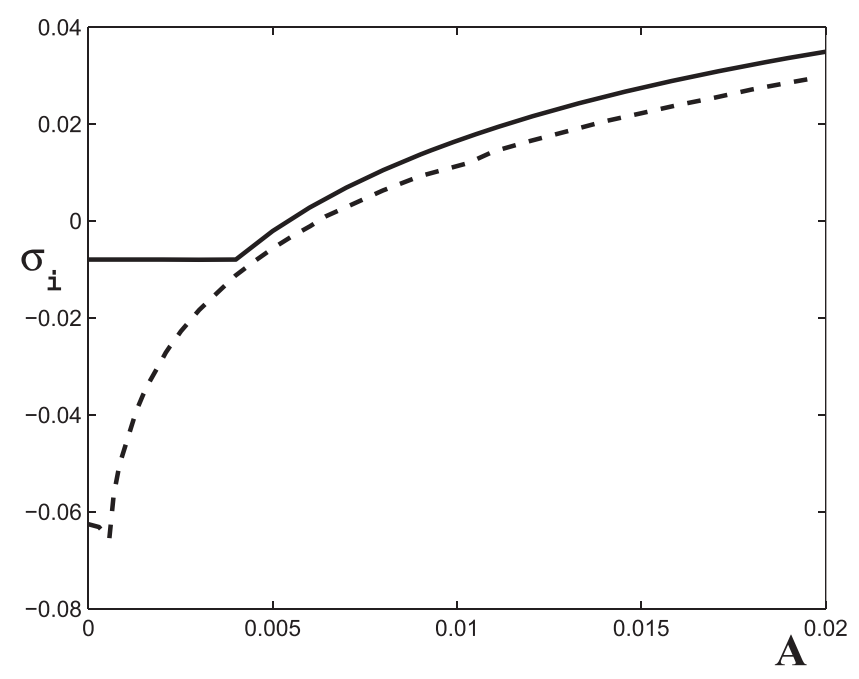

FIG. 8. Amplification rate for the dominant mode of subharmonic instability as a function of $\beta$ at $R e=5000$ and $\alpha=1.12$. Solid and dashed curves represent present and Herbert's ${ }^{2}$ results.

is also shown. Again, though the overall shape is the same for higher amplitudes, there is a discrepancy at lower amplitudes because a different mode becomes dominant as $A \rightarrow 0$, as described earlier. However, Herbert continues to track the mode that was dominant for larger $A$. That is why the present curve has a slope discontinuity for $A \approx 0.004$, whereas the dashed curve is continuous.

We now discuss convergence of the solutions. The spectra shown in Figs. 3-5 have not converged. This can be simply seen by increasing the number of Fourier and Chebyshev modes $M$ and $N$. The convergence of the full spectrum with respect to $N$ is shown in Fig. 9, for $M=2$. It can be seen that $N=100$ is enough for convergence, at the given $M$. The eigenvalue corresponding to the most unstable mode was tracked as $M$ is increased, for different $N$. The results are shown in Table I. A computation with $M=3, N=100$ produced a value of $0.00181+0.07803 i$ which shows a converging trend, when compared with the values in the table. The difference between this eigenvalue and that of Herbert's ${ }^{2}$ is of the order of $10^{-2}$, which becomes quite significant if threshold amplitudes for secondary growth have to be estimated from this eigenvalue.

\section{B. Threshold amplitudes of the subharmonic and fundamental disturbances}

Herbert (Ref. 2) lists the minimum subharmonic and fundamental threshold amplitudes, defined as the amplitudes below which the modes are stable, of $A=0.0042$ at $\beta=0.74$ and $A=0.0085$ at $\beta=1.22$ for $\alpha=1.02$. Figures 10 (a) and 10(b) show the variation of threshold amplitudes of subharmonic and fundamental disturbances with $\beta$ at the same $\alpha=1.02$. Instability regions are shown shaded, with the boundary separating the black and white regions marking the threshold amplitudes for a given $\beta$. The subharmonic diagram has no reentrant points, i.e., for a given $\beta$, there is only one threshold beyond which the flow is unstable. The threshold values also exhibit only one minimum. The present subharmonic threshold values of $A_{s} \approx 0.0036$, occurring at $\beta_{s} \approx 0.74$, compare favorably with Herbert's. $^{2}$ The fundamental case shows more complexity. 


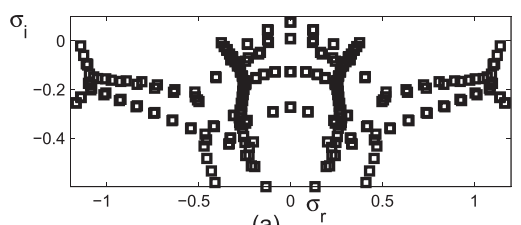

(a)

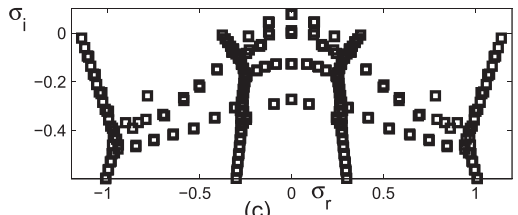

(c)

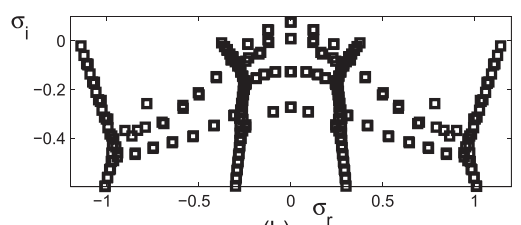

(b)

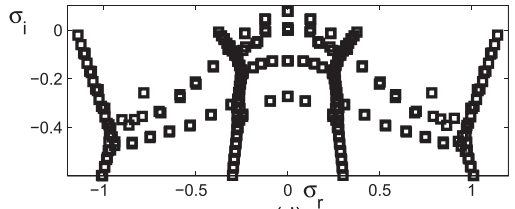

(d)
FIG. 9. Temporal subharmonic spectrum for $\alpha=1.12, \beta=2, R e=5000$, and $A=0.0248$. The number of Fourier modes, $M=2$. The number of Chebyshev $\operatorname{modes} N=$ (a) 50 , (b) 100 , (c) 150 , (d) 200 .
TABLE I. Convergence of the most unstable subharmonic mode with respect to $M$ and $N$. $R e=5000, \alpha=1.12, \beta=2, A=0.0248$.

\begin{tabular}{lcc}
\hline \hline & & $\mathrm{N}$ \\
\cline { 2 - 3 } $\mathrm{M}$ & 50 & 100 \\
\hline 1 & $0.00305+0.06119 i$ & $0.00304+0.06113 i$ \\
2 & $0.00196+0.07703 i$ & $0.00196+0.07691 i$ \\
\hline $\mathrm{M}$ & 150 & 200 \\
\hline 1 & $0.00304+0.06112 i$ & $0.00304+0.06112 i$ \\
2 & $0.00196+0.07688 i$ & $0.00196+0.07689 i$ \\
\hline \hline
\end{tabular}

For a narrow wavenumber band $\beta \approx 0.49-0.56$, stable and unstable regions alternate for a range of amplitudes. The thresholds also exhibit multiple minima and even a local maximum. The lowest fundamental threshold values are $A_{f} \approx$ 0.0035 , occuring at $\beta_{f} \approx 0.21$; these are quite different from Herbert's (Ref. 2) values given earlier. Intriguingly, one of the minima does occur at $A_{H} \approx 0.0071, \beta_{H} \approx 1.25$ whose values are close to Herbert's. However, these are not the global minima, as Herbert seems to suggest.

Similar diagrams can be generated from half-channel computations as well. In the case of the subharmonic modes, there is no difference in the full-channel and half-channel computations, as the distinction between even and odd modes is redundant ${ }^{8}$ as far as threshold computations are concerned. However, the real parts of the eigenvalues of the two families will be negative of one another. The eigenfunctions will be different as well, one being even and the other being odd.

The fundamental case is quite different. The presence of an aperiodic term in the velocity expansions leads to different stability diagrams depending on which set of eigenvalues is used to generate the diagrams. Figure 11 shows the thresholds from a half-domain computation. Figures 11(a) and 11(b) correspond to the cases $C_{a}$ and $C_{s}$ where the aperiodic term $u_{0}$ is antisymmetric (symmetric), respectively. Though the composite of the two produces the threshold of Fig. 10(b), the individual threshold diagrams are different, unlike in the subharmonic case. Thus, if one were to confine oneself to the case $C_{a}$, like Herbert ${ }^{2}$ did, one would get $A_{H}$ and $\beta_{H}$ close to Herbert's values, as stated earlier. The case $C_{s}$ however produces the lower global threshold, also stated earlier. Herbert (Ref. 2) seems to have overlooked this component. He does state in Ref. 8, and we slightly paraphrase-_The curious properties of the symmetric fundamental mode at low amplitude and small $\beta$ will be discussed elsewhere." However, we are not aware of such a discussion. Moreover, for $\beta \in \approx[0.48,1.2]$, the unstable mode has a phase velocity different from that of the TS wave. This reflects the fact that the unstable mode is off the imaginary axis for this range of $\beta$. Figure 12 shows the eigenfunctions corresponding to the lowest thresholds from each of the cases $C_{a}\left(A_{H}=0.0071, \beta_{H}=1.25\right)$ and $C_{s}\left(A_{f}=0.0035, \beta_{f}=0.21\right)$, respectively.

Figure 13 shows the threshold amplitudes for a variety of detuned resonances, when $\gamma$ varies between the fundamental and subharmonic cases. Some of these have amplitude thresholds that are even lower than the fundamental one. The

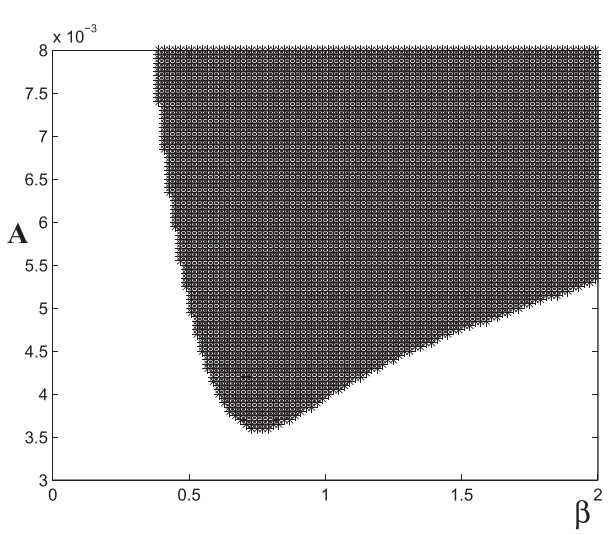

(a)

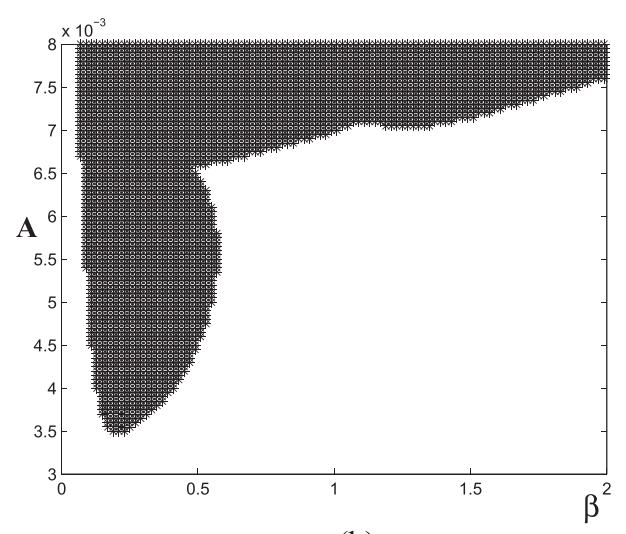

(b)
FIG. 10. Threshold amplitudes, as a function of a spanwise wavenumber $\beta$ for (a) subharmonic modes and (b) fundamental modes. Note that the lowest fundamental threshold is lower than the lowest subharmonic one, in contradiction with Herbert's (Ref. 2) claim. 


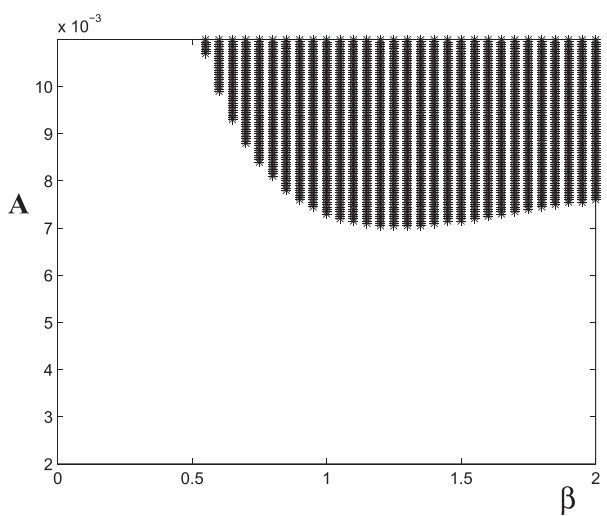

(a)

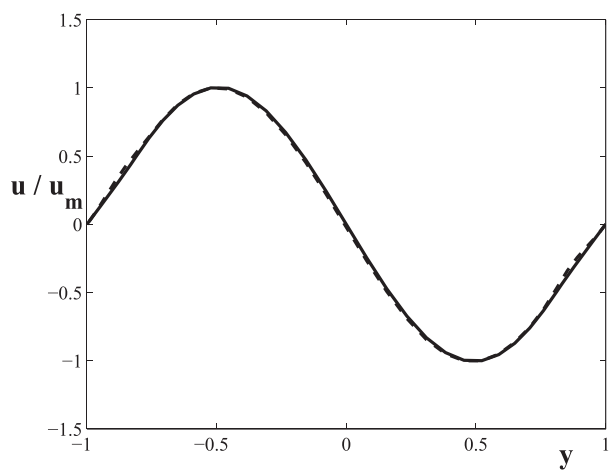

(a)
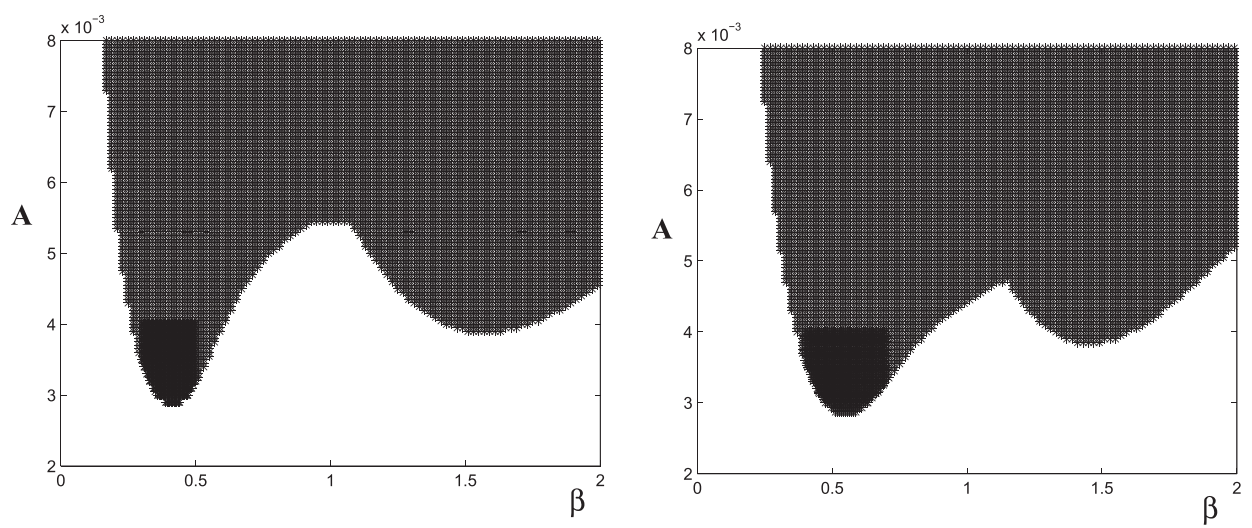

(a)

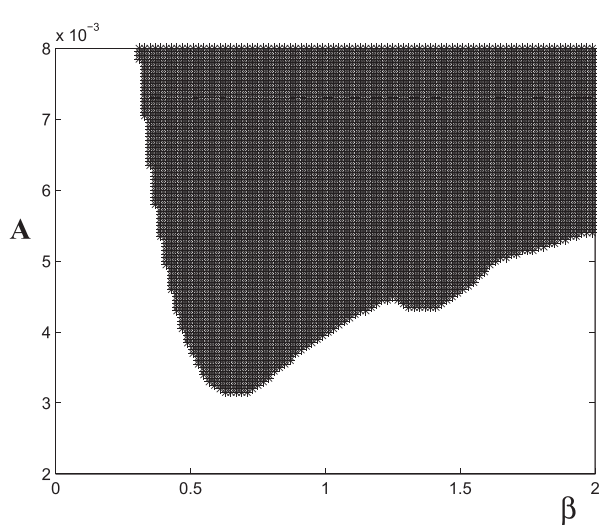

(b)

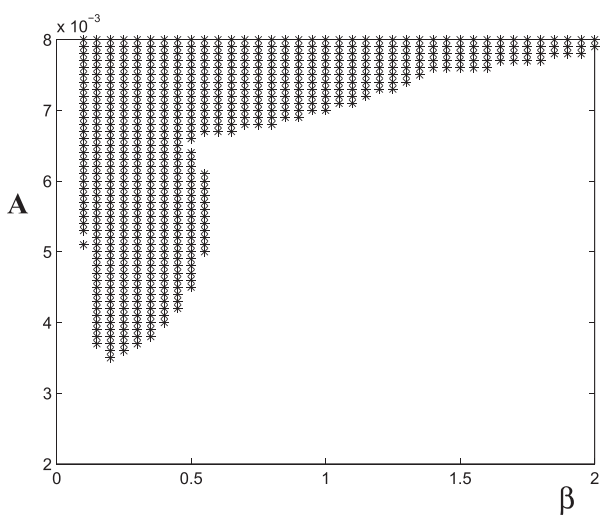

(b)

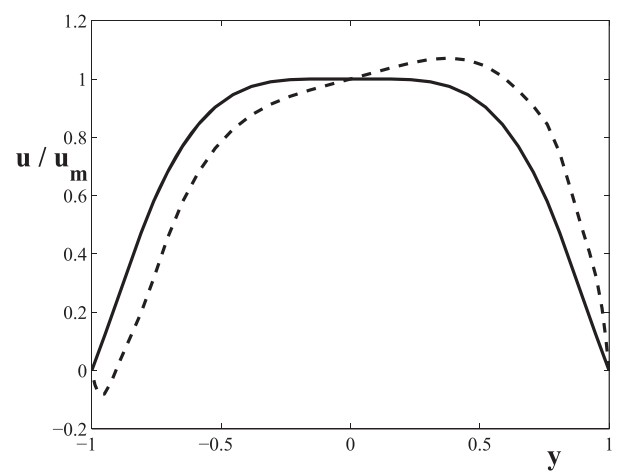

(b)

FIG. 11. Threshold amplitudes, as a function of a spanwise wavenumber $\beta$ for the (a) odd and (b) even components of the fundamental modes, from half domain computations with appropriate boundary conditions. Note that the odd fundamental threshold is in fair agreement with Herbert's (Ref. 2) value, whereas the even threshold is actually the global minimum and apparently overlooked by Ref. 2 .

FIG. 12. Fundamental eigenfunctions for (a) antisymmetric case $\left(A_{t}=0.0071\right.$, $\beta=1.25)$, (b) symmetric case $\left(A_{t}=\right.$ $0.0035, \beta=0.21$ ). Solid lines represent the aperiodic component, $u_{0}$.

FIG. 13. Threshold amplitudes, as a function of a spanwise wavenumber $\beta$ for detuned modes. The Floquet exponent $\gamma=$ (a) $0.125 i$, (b) $0.25 i$, (c) $0.375 i$. The fundamental and subharmonic modes have $\gamma=0$ and $\gamma=$ $0.51 i$.

(c) 


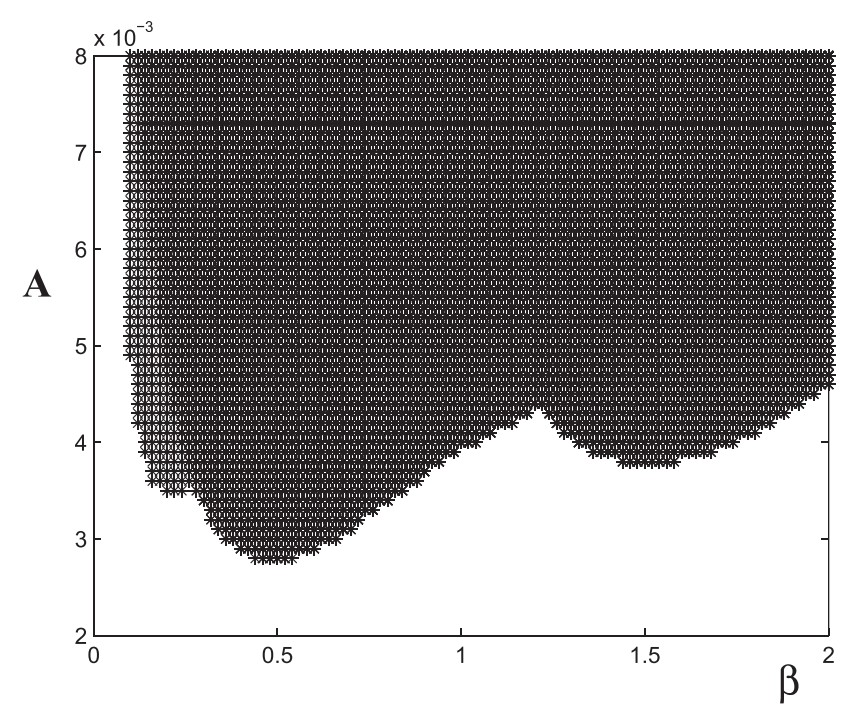

FIG. 14. Composite stability diagram, computed over all modes ranging from the fundamental to the subharmonic. $R e=5000$ and $\alpha=1.02$.

fundamental and detuned modes show two minima: a sharp one at lower $\beta$ and a broader one at higher $\beta$, with the sharp one being the smaller of the two. A local maximum is also exhibited for these resonances. Around $\gamma \approx 0.4 i$, the local maximum and the higher minimum coincide; beyond this value, only the lower $\beta$ minimum exists with the threshold being monotonic on either side.

Figure 14 presents a composite of the threshold amplitudes, obtained over all $\gamma \in[0,0.51 i]$ and $\beta \in[0.1,2]$. The composite profile demonstrates three minima, the first due to the fundamental mode and the other two being produced by a detuned mode with $\gamma \approx 0.2$.

Figure 15 presents stability diagrams analogous to Fig. 10, but computed with $M=2$ and $N=160$. The shape of the threshold amplitude envelope remains substantially the same as that computed with $M=1$ and $N=40$; the values differ a little as noted earlier.

\section{Comparison of disturbance velocity profiles}

A key component of the comparison between theory and experiment and between different calculations is the shape of the disturbance velocity profile. While the theoretical calculations can provide the various Fourier components $u_{m}, v_{m}$

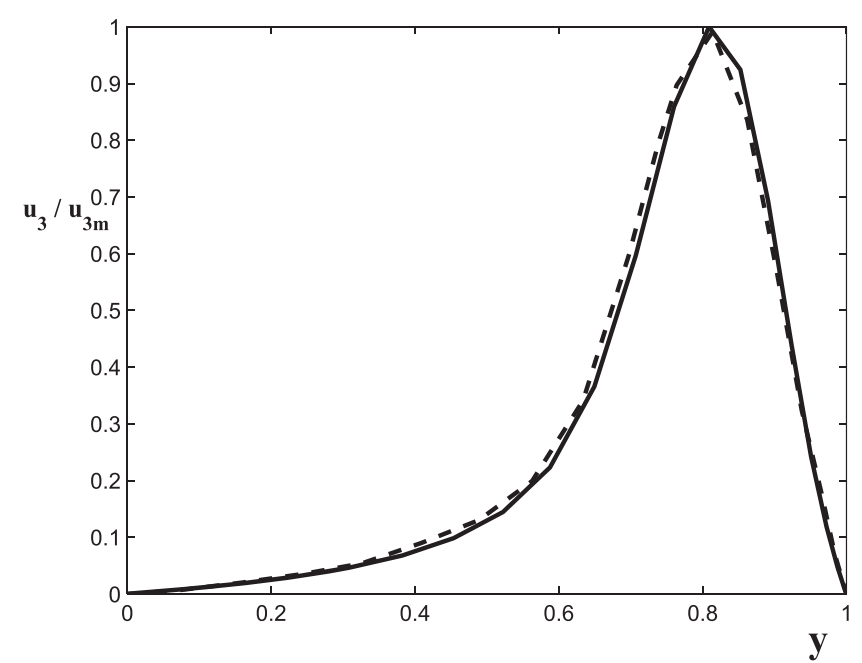

FIG. 16. Streamwise secondary disturbance velocity for the subharmonic mode at $R e=5000, \alpha=1.12, \beta=2$ and $A=0.0248$. Solid and dashed curves represent present and Herbert's (Ref. 2) results.

[Eq. (3)], the total disturbance velocity depends on the moving frame coordinate $\xi$. The experimentalists, on the other hand, measure the total disturbance velocity in the lab reference frame and have to extract the particular Fourier component of interest for comparison.

We start with a comparison with Herbert's ${ }^{2}$ results for the subharmonic case considered earlier $(\operatorname{Re}=5000, \alpha=1.12$, $\beta=2, A=0.0248$ ). Figure 16 shows the present computation of the streamwise disturbance component $u_{3}$ normalised with its maximum value along with Herbert's calculation of the same. We have assumed the moving coordinate $\xi=0$. The comparison is good.

Figure 17 shows a comparison for the fundamental symmetric mode, which is the dominant mode at the considered parameter value. The solid line represents the present computation of the fundamental Fourier component $\left[u_{1}\right.$ in Eq. (3)] with $M=2$, while the dashed-dotted line (almost indistinguishable from the solid) is with $M=1$. The dashed line is the total fundamental streamwise disturbance component obtained from Fig. 1 of Ref. 8, after subtracting the TS part. The circles represent experimental values, synthesised from the amplitude and phase measurements of the study of Nishioka and Asai. ${ }^{9}$ It is important to note several points about

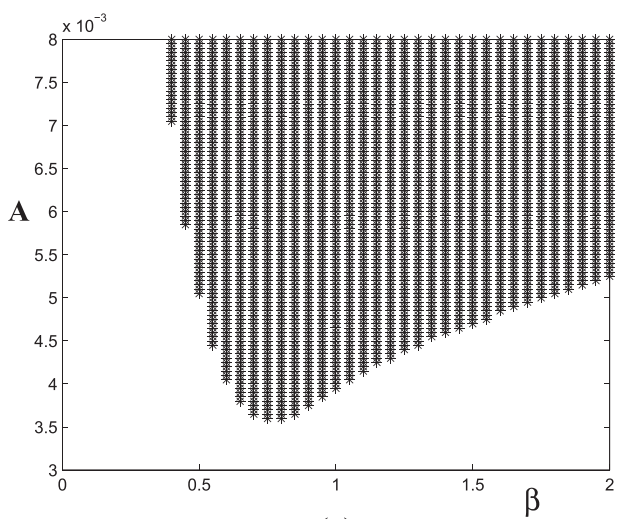

(a)

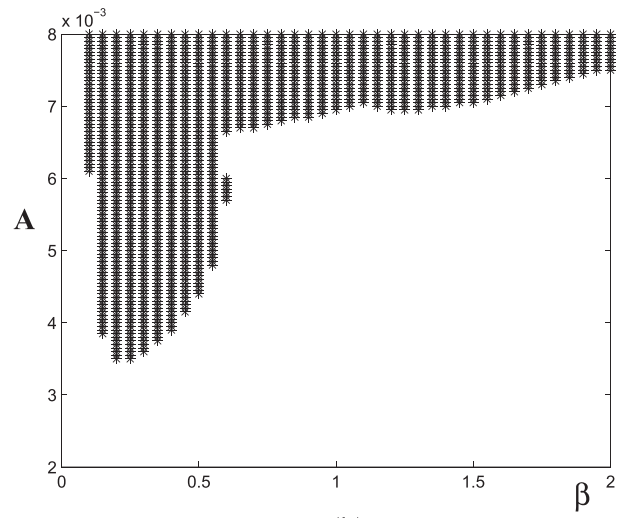

(b)
FIG. 15. Threshold amplitudes, as a function of a spanwise wavenumber $\beta$ for (a) subharmonic modes and (b) fundamental modes. Two Fourier and 160 Chebyshev modes are used. 


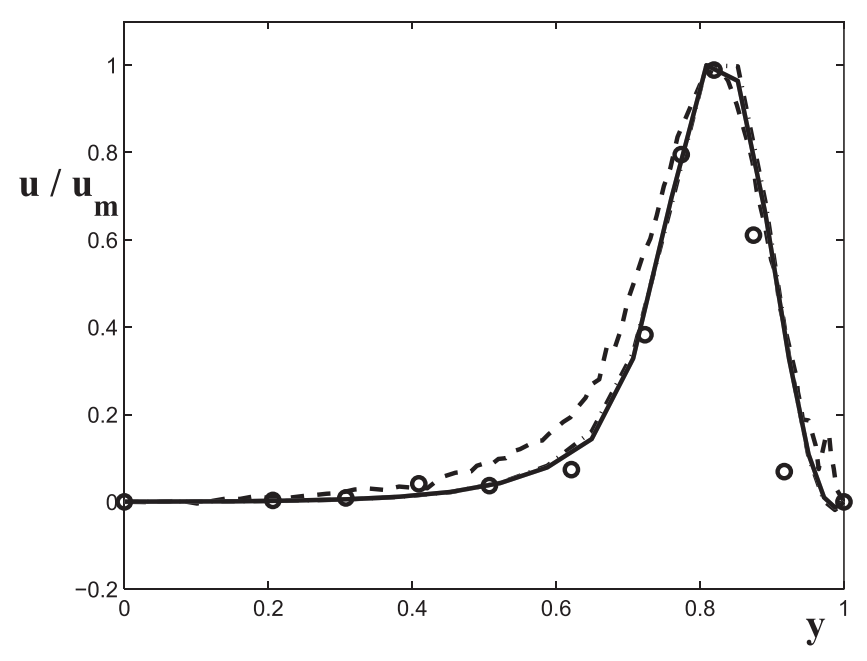

FIG. 17. Streamwise secondary disturbance velocity for the even fundamental mode. The solid line and the circles, which represent experimental values of the study of Nishioka and Asai, ${ }^{9}$ are for $\operatorname{Re}=5000, \alpha=1.16, \beta=1.84$, and $A=0.018$. The dashed line represents the computation by Herbert (Ref. 8 ) for $\operatorname{Re}=5000, \alpha=1.12, \beta=2$, and $A=0.0248$.

the quantities in this figure. We infer from the study of Nishioka and Asai $^{9}$ that their experimental profile is of the fundamental Fourier component; the curve representing the present computation is for the same component as well. On the other hand, we also infer from Herbert ${ }^{8}$ that his curve represents the total fundamental streamwise disturbance, not just the fundamental Fourier component. Also, the Herbert computation is for $\alpha=1.12, \beta=2$, and $A=0.0248$, whereas the present computation is for $\alpha=1.16, \beta=1.84$, and $A=0.018$, same as the experimental values. Nishioka and Asai ${ }^{9}$ claim good comparison with Herbert's curve. However, it is clear from the figure that the comparison is better with the present computation of the fundamental Fourier component of the streamwise disturbance.

\section{DISCUSSION}

It has been widely held for atleast 30 years that three amplitude ranges, of the basic 2D TS wave, can be distinguished in $\mathrm{pPf}$ - the lowest range with no 3D instability, an intermediate range with instability to only subharmonic disturbances and a large amplitude range where the flow is unstable to both harmonic and subharmonic disturbances (for example, p. 286 of the study of Godreche and Mannville ${ }^{10}$ ). However, no signatures of the subharmonic modes have been experimentally reported even at the lowest TS amplitudes.

This discrepancy was sought to be explained by DNS studies of natural transition, such as Kim and Moser ${ }^{11}$ and Zang and Krist. ${ }^{12}$ One explanation was that the spanwise modes were preferentially elevated above the background level and a nonlinear interaction between these and the primary wave led to a growth of the fundamental $[(1,1)$ or $(1,2)$ in these studies] such that these modes were present at higher amplitudes than say the subharmonic so that even with the latter modes starting to grow at lower primary amplitudes, it was the former which, by dint of their initial higher values, were actually seen in the experiments.

It may be noted however that the "fundamental mode" of these studies is not the same as that of the Floquet analysis of Herbert; the latter's fundamental has many Fourier components, one of which is the former and another is the spanwise mode. It is also important to note that all these components evolve temporally at the same rate. One way the subharmonic mode could appear was if it was initially seeded to be of comparable amplitude to the fundamental and in fact, staggered lambda patterns ${ }^{13}$ (corresponding to subharmonic modes) and even detuned modes ${ }^{14}$ have been observed in boundary layer flows by precisely such seeding. The sole observation of staggered patterns in pPf is due to Ramazanov, ${ }^{15}$ who also had to seed the subharmonic mode. However, most of the experimental observations, of staggered and peak-valley structures, and of streamwise velocity disturbance profiles, are at high primary disturbance amplitudes.

The present study shows that the symmetric fundamental modes can have a lower or comparable threshold amplitude to the subharmonic and thus presents another scenario for the possible appearance of the fundamental. A second possibility is that slightly detuned modes, which may have lower thresholds than even the fundamental but whose velocity profiles may not be very different, are the ones that are actually seen in the experiments. A confirmation of these would require experimental observations at lower disturbance amplitudes than have been reported in the literature.

Crouch and Herbert ${ }^{16}$ considered the weakly nonlinear evolution of secondary disturbances in boundary-layer transition. Such an analysis requires the identification of the dominant secondary mode(s) as the primary disturbance amplitude tends to zero. If a similar analysis is performed for the pPf and surprisingly it does not seem to have been done till now, the present findings would be of value in such an identification.

\section{CONCLUDING REMARKS}

We have shown that there does not exist, in pPf, a low TS amplitude range in which only subharmonic modes become unstable, contrary to the prevailing view. Moreover, we have found that the amplitude thresholds of some detuned modes can be even lower than the fundamental's for some $\beta$. The fact that the fundamental threshold can be lower than the subharmonic provides a possible alternative explanation, different from that given, for example, in the study of Kim and Moser ${ }^{11}$ for the preponderance of the fundamental instability, even at low disturbance amplitudes, contrary to the predictions of Herbert $^{1,2}$ that the subharmonic instability should dominate at these amplitudes.

These new modes would also have to be considered as possible candidates for a weakly nonlinear analysis of the secondary instability in plane Poiseuille flow. The present study makes detailed predictions, which include shapes of eigenfunctions (Fig. 12), about the possible fundamental instability at very low disturbance amplitudes; these could be tested by new experimental observations at these disturbance amplitudes. 


\section{ACKNOWLEDGMENTS}

The authors acknowledge the financial support from National Board of Higher Mathematics, Department of Atomic Energy, India [Project No. 2/48(3)/2013/NBHM(RP)/ R\&DII/685].

\section{APPENDIX: SECONDARY DISTURBANCE EQUATIONS}

Substituting (2) into the N-S equations, linearising in $\epsilon$, eliminating the pressure and the spanwise velocity component $w_{3}$ by taking curl and using continuity, respectively, a coupled PDE system for the streamwise and normal velocity components $u_{3}$ and $v_{3}$ is obtained as in the study of Herbert et al. ${ }^{5}$

$$
\begin{aligned}
& {\left[\frac{1}{R e} \nabla^{2}-\left(U_{0}-c\right) \frac{\partial}{\partial x}-\frac{\partial}{\partial t}\right] \frac{\partial \eta_{3}}{\partial z}+\zeta_{0} \frac{\partial^{2} v_{3}}{\partial z^{2}}} \\
& +A\left[\left(-\frac{\partial \psi_{1}}{\partial y} \frac{\partial}{\partial x}+\frac{\partial \psi_{1}}{\partial x} \frac{\partial}{\partial y}-\frac{\partial^{2} \psi_{1}}{\partial x \partial y} \frac{\partial \eta_{3}}{\partial z}\right.\right. \\
& \left.+\frac{\partial^{2} \psi_{1}}{\partial x^{2}}\left(\frac{\partial^{2} u_{3}}{\partial x \partial y}+\frac{\partial^{2} v_{3}}{\partial y^{2}}\right)-\frac{\partial^{2} \psi_{1}}{\partial y^{2}} \frac{\partial^{2} v_{3}}{\partial z^{2}}\right]=0 \\
& {\left[\frac{1}{R e} \nabla^{2}-\left(U_{0}-c\right) \frac{\partial}{\partial x}-\frac{\partial}{\partial t}\right] \nabla^{2} v_{3}-\frac{d \zeta_{0}}{d y} \frac{\partial v_{3}}{\partial x}} \\
& +A\left[\left(-\frac{\partial \psi_{1}}{\partial y} \frac{\partial}{\partial x}+\frac{\partial \psi_{1}}{\partial x} \frac{\partial}{\partial y}\right) \nabla^{2} v_{3}+\frac{\partial^{2} \psi_{1}}{\partial x^{2}}\left(\frac{\partial \zeta_{3}}{\partial y}+\frac{\partial \eta_{3}}{\partial z}\right)\right. \\
& \quad-\frac{\partial^{2} \psi_{1}}{\partial x \partial y}\left(\frac{\partial \zeta_{3}}{\partial x}+\frac{\partial \xi_{3}}{\partial z}\right)-\frac{\partial \zeta_{1}}{\partial x}\left(2 \frac{\partial u_{3}}{\partial x}+\frac{\partial v_{3}}{\partial y}\right) \\
& \left.\quad-\frac{\partial \zeta_{1}}{\partial y} \frac{\partial v_{3}}{\partial x}-\left(u_{3} \frac{\partial}{\partial x}+v_{3} \frac{\partial}{\partial y}\right) \frac{\partial \zeta_{1}}{\partial x}\right]=0
\end{aligned}
$$

(A1) and (A2) have to be solved subject to the boundary conditions $u_{3}=v_{3}=\partial v_{3} / \partial y=0$ at $y= \pm 1$.
${ }^{1}$ Th. Herbert, "Secondary instability of boundary layers," Annu. Rev. Fluid Mech. 20, 487-526 (1988).

${ }^{2} \mathrm{Th}$. Herbert, "Secondary instability of plane channel flow to subharmonic three-dimensional disturbances," Phys. Fluids 26(4), 871-874 (1983).

${ }^{3}$ S. A. Orszag and A. T. Patera, "Secondary instability of wall bounded shear flows," J. Fluid Mech. 128, 347-385 (1983).

${ }^{4}$ P. J. Schmid and D. S. Henningson, Stability and Transition in Shear Flows, Applied Mathematical Sciences (Springer, 2001), Vol. 142.

${ }^{5}$ Th. Herbert, F. P. Bertolotti, and G. R. Santos, "Floquet analysis of secondary instability in shear flows," in Stability of Time Dependent and Spatially Varying Flows: Proceedings of the Symposium on the Stability of Time Dependent and Spatially Varying Flows Held on 19-23 August 1985 at NASA Langley Research Center, Hampton, Virginia, edited by D. L. Dwoyer and M. Y. Hussaini (Springer-Verlag, 1987), pp. 43-57

${ }^{6}$ Hydrodynamic Instability and Transition to Turbulence, Fluid Mechanics and Its Applications, edited by A. M. Yaglom and U. Frisch (Springer, 2012), Vol. 100.

${ }^{7}$ A. H. Nayfeh and D. T. Mook, Nonlinear Oscillations (John Wiley \& Sons, 1995).

${ }^{8}$ Th. Herbert, "Modes of secondary instability in plane Poiseuille flow," in Turbulence and Chaotic Phenomena in Fluids: Proceedings of the International Symposium on Turbulence and Chaotic Phenomena in Fluids Held on 5-10 September 1983 at Kyoto, Japan, edited by T. Tatsumi (North-Holland, 1983), pp. 53-58.

${ }^{9}$ M. Nishioka and M. Asai, "Three-dimensional wave-disturbances in plane Poiseuille flow," in Laminar-Turbulent Transition: IUTAM Symposium, edited by V. V. Kozlov (USSR, Novosibirsk, 1984), pp. 173-182.

${ }^{10} \mathrm{C}$. Godreche and P. Manneville, Hydrodynamics and Nonlinear Instabilities (Cambridge University Press, 2005).

${ }^{11}$ J. Kim and R. D. Moser, "On the secondary instability in plane Poiseuille flow," Phys. Fluids A 1(5), 775-777 (1989).

${ }^{12}$ T. A. Zang and S. E. Krist, "Numerical experiments on stability and transition in plane channel flow," Theor. Comput. Fluid Dyn. 1, 41-64 (1989).

${ }^{13}$ W. S. Saric and A. S. W. Thomas, "Experiments on the subharmonic route to turbulence in boundary layers," in Turbulence and Chaotic Phenomena in Fluids: Proceedings of the International Symposium on Turbulence and Chaotic Phenomena in Fluids Held on 5-10 September 1983 at Kyoto, Japan, edited by T. Tatsumi (North-Holland, 1983), pp. 117-122.

${ }^{14}$ Yu. S. Kachanov and V. Ya. Levchenko, "The resonant interaction of disturbances at laminar-turbulent transition in a boundary layer," J. Fluid Mech. 138, 209-247 (1984).

${ }^{15}$ M. P. Ramazanov, "Development of finite-amplitude disturbances in Poiseuille flow," in Laminar-Turbulent Transition: IUTAM Symposium, edited by V. V. Kozlov (USSR, Novosibirsk, 1984), pp. 183-190.

${ }^{16} \mathrm{~J}$. D. Crouch and T. Herbert, "Nonlinear evolution of secondary instabilities in boundary-layer transition," Theor. Comput. Fluid Dyn. 4, 151-175 (1993). 